\title{
A Review on Applications of Nanobots in Combating Complex Diseases
}

\author{
Tashnuva Rifat, Md. Shahadat Hossain, Md. Mahbubul Alam and \\ Abu Shara Shamsur Rouf
}

\author{
Department of Pharmaceutical Technology, Faculty of Pharmacy, University of Dhaka \\ Dhaka-1000, Bangladesh
}

(Received: September 21, 2018; Accepted: December 31, 2018; Published: January 17, 2019)

\begin{abstract}
In the current years, a lot of research works have been continued in the quest to find a desired drug delivery system within the human body. Among different drug delivery systems, nanobots have become much popular due to its capability to perform different tasks like actuating, sensing, signaling, information processing and intelligence at nanoscale dimension. A nanobot is a robotic machine that can be programmed to diagnose, monitor and treat various vital diseases. It works at cellular level with minimum side effects and thus substitute the conventional treatment procedures. The major limitations of nanobots are proper designing and manufacturing process. In spite of its expensive manufacturing set up, they are pre-eminent for medical applications, such as nanobots like respirocytes, microbivores and clottocytes have been designed to act as artificial substitutes of blood. This article describes the use of nanobots in different fields of medical science like diagnosis and treatment of cancer, diabetes, aneurysm, tetanus, HIV, skin disease, gout as well as in the field of dentistry, surgery and treating many other diseases. It also represents the recent research which has been applied in vivo to different animals. Scientists hope that nanobots will act on molecular level, protect the biological system and thus ensure a better and longer life.
\end{abstract}

Key words: Nanobot, current research works, respirocyte, microbivore, clottocyte.

\section{Introduction}

The word like nanobot or nanorobot has captured public imagination over the last two decades. This is a machine of nanoscale dimension that can be viewed under microscope. Such devices play an essential role in critical care applications (Nerlich, 2008). Nanorobotics is a part of nanotechnology that deals with the study of designing, programming, manufacturing and controlling of robots at nanoscale which ranges from 0.1 to $10 \mu \mathrm{m}$. A nanobot is an extremely small robot that has been designed to perform a specific task with precision at nanoscale. These are also known as nanorobots, nanites, nanoagents and nanoids (Manjunath and Kishore, 2014).
The word nanobot was first used in 1989 to designate a microscopic robot used in nanotechnology. A nanobot is an extremely small autonomous self-propelled machine. According to web-dictionary for nanoscience and nanotechnology "A nanobot is an imaginary machine on a scale of few to few hundreds of nanometers designed to perform specific tasks". The prototype models for most of the futuristic concepts are specific cells e.g. phagocytes which ingest foreign matters and cellular molecular machineries like RNA polymerase or ribosome (Nanobot, Websters New Millennium ${ }^{\mathrm{TM}}$ Dictionary of English).

Nanobots are designed from the biological model of bacteria. The principle element used in

Correspondence to: Abu Shara Shamsur Rouf; Email: rouf321@ yahoo.com 
construction of nanobot is carbon and it is comprised probably in the form of carbon or fullerence nano composites. Due to the inert properties, high thermal conductivity and strength of diamondoid material, it is used in the construction of the outer shell of the nanobot, while the super smooth surface also helps to reduce the chance of triggering the body's immune system. The mechanical parts by which a nanobot is made of are bearing, gears, motors etc., where the nanoscale gears and other components can be constructed using elements like hydrogen, sulfur, oxygen, nitrogen or silicon. Moreover, the substructures of nanobot involve onboard power supply, sensors, nanocomputer, pumps, manipulators and pressure tanks (Uriarte, 2011).

Applications of nanobots in medicine provide a new range of tools for the treatment of diseases and the improvement of human biological system. The design of various nanobots in medicine includesrespirocytes, microbivores, clottocyes, pharmacytes, dentifrobots and vasculoids (Freitas, 2007).

Due to its minimum side effects and quicker response, nanobot is found to be more innovative and supportive to the treatment and diagnosis of severe diseases like cancer, heart attack, diabetes, atherosclerosis, kidney stones etc (Robert, 2009). Through the ability for regular monitoring of body condition, nanobots allow us a personalized treatment and thus achieve high efficiency against many diseases (Manjunath and Kishore, 2014). For all of these reasons nanobots are going to introduce a new chapter in the field of medical science.

This article mainly focuses on the applications of nanobots in different fields of medical science e.g. hematology, cancer, dentistry, diabetes, heart diseases, gene therapy, skin diseases, surgery etc. It also contains the information of different research studies on nanobots along with the advantages over the conventional treatment procedures, which will ultimately help the researchers to design and conduct further research on nanobots.

Applications of nanobots in medical science: Medical nanobots can be used in diagnosis, monitoring and treating critical diseases. These are capable of delivering medicine into the specific target site in human body. The potential applications of nanobots include:

Hematology: Nanobots have potential application in the field of hematology. Its use in hematology ranges from emergency transfusions of non-blood oxygen carrying compounds to restoring primary hemostasis (Saadeh and Vyas, 2014)

Respirocytes are spherical $1 \mu \mathrm{m}$ diameter sized nanobots which are designed as artificial mechanical red blood cells. The respirocyte could deliver 236 times more oxygen to the body tissue when compared to natural red blood cells. The respirocyte would manage the carbonic acidity that can be controlled by gas concentration sensors and on-board nanocomputer (Freitas, 2005a). Each respirocyte has 3 types of rotors. One rotor releases the stored oxygen while travelling through the body. The second rotor captures all the carbon dioxide at blood stream and releases at the lungs. The third rotor takes glucose from blood stream and uses as fuel source (Mishra and Dash, 2012). It can be programmed to scavenge carbon monoxide and other poisonous gases from the body. A $5 \mathrm{cc}$ therapeutic dose of 50\% respirocyte saline suspension contains 5 trillion nanorobots that could exactly replace the gas carrying capacity of the patient's entire 5.4 litres of blood (Freitas, 2005b).

Microbivores are the nanobots that are designed as artificial WBC and also known as nanobotic phagocytes. It is a spheroid device made of diamond and sapphire which measures $3.4 \mu \mathrm{m}$ in diameter along its major axis and $2 \mu \mathrm{m}$ diameter along its minor axis. Microbivore absorbs and digests the pathogens in the blood stream by the process of phagocytosis (Eshaghian-Wilner, 2009). During the cycle of operation, the target bacterium binds to the microbivore surface via specific reversible binding site. A collision between the bacterium and microbivore brings in the surface into close contact and thus the reversible binding site can be recognized and weakly bound to the bacterium. When the bacterium is bound to the binding site, the telescopic 
robotic grapples rise up from the surface and attach to the bacterium. Then the bacterium is transported from the binding site to the injection port by the grapple's handoff motion. Then the bacterium is internalized into the morcellation chamber where the bacterium is minced into nanoscale pieces. These pieces of bacterium are pistoned into digestive chamber which consists of pre-programmed set of digestive enzymes. Then it is converted into amino acids, mononucleotides, free fatty acids and simple sugars which are then discharged into blood stream through the exhaust port. It needs 30 seconds to complete the entire cycle of phagocytosis by microbivore (Manjunath and Kishore, 2014). Microbivore acts 1000 times faster than antibiotic aided WBCs and the pathogen stand no chance of multiple drug resistance which occurs in case of antibiotic. They can also be used in case of respiratory and cerebrospinal bacterial infection or infection in urinary fluid and synovial fluid (Eshaghian-Wilner, 2009).

Clottocyte is a nanobot that is designed as artificial platelets. It would complete hemostasis within 1 second. The response time is 100-1000 times faster than the natural hemostatic system which takes 2-5 minutes to complete the whole process (Boonrong and Kaewkamnerdpong, 2011). The application of nanobots as clottocytes has reduced the clotting time and blood loss. Again, blood clots are found to occur irregularly in some patients. This abnormality is treated by corticosteroids which is associated with side effects such as hormonal secretion, lung damage and allergic reaction. Clottocytes can be used as an alternative treatment which is free from these side effects (Manjunath and Kishore, 2014).

Dentistry: The nanobots designed to apply in dental treatment are known as dentifrobots (Abhilash, 2010). These are used in routine cleaning, tooth whitening, hypersensitivity and even in orthodontics (Sahoo et al., 2007).

A mouth wash and toothpaste full of nanobots can identify and destroy the pathogenic bacteria while allow the harmless flora of mouth. These devices can identify food particles, plaque or tartar and can remove them effectively. Nanobots provide complete dentition replacement therapy including both mineral and cellular components. Orthodenticnanobots can manipulate the periodontal tissue including periodontal ligament, gingiva, alveolar bone and cementum (Sujatha et al., 2010). Nanobots can be covered by highly specific proteins that bind to the targeted pathogens for the treatment of infection. In case of root canal, the use of tiny camera can provide visualization of root and thus reduces any guess work. In hypersensitive teeth, the number and diameter of dentinal tubules are significantly increased, where penetration of nanobotswith selective ablation or occlusion ability can prevent these stimuli within minute (Sharples, 2011).

Cancer detection and treatment: Cancer can be successfully diagnosed and treated with the help of nanobots. Unlike the conventional drug, nanobots are highly site specific i.e. it is programmed to detect only the diseased cells to act upon them, the healthy cells are left aside and thus show minimum side effects. Nanobots with embedded biosensors can be used in detection of tumor cells in the early stage of development inside the patient's body (Sivasankar and Durairaj, 2012). Kumar et al. (2014) has reported that, scientists have genetically modified salmonella bacteria carrying microscopic robots $(3 \mu \mathrm{m})$ named bacteriobots, which are drawn to tumors by chemicals secreted by the cancerous cells. These deliver drug directly to the tumor leaving the healthy cells alone and thus protect the patient from side effects of chemotherapy. But these bacteriobot can only detect the tumor in case of breast cancer and colorectal cancers. On the contrary, nanobot is able to detect and treat other cancers (Kumar et al., 2014)

This nanobot can be constructed to different cell surface receptors. The payload it releases upon activation, can also be changed as necessary. The nanobot is constructed using engineered DNA strands that have been made to fold into a desired tertiary structure (Dietz et al., 2009). After binding to the desired target, the conformation of the DNA nanobot 
changes from closed state to tertiary state to release the stored therapy (Douglas et al., 2012).

Murnanehas reported that nanobots can combat the tumor by preventing the flow of blood to the tumor cells. As without blood flow tumor cells cannot survive, the researchers have targeted this weak point. This nanobot is made from a flat DNA sheet to which an enzyme thrombin is attached which is responsible for blood clotting. This sheet is then rolled into a tube with thrombin inside (Murnane, 2018). DNA aptamers are attached to the surface of the tube that seeks out a protein called nucleoin that present in the endothelial cells on the surface of only the tumor cells not on the surface of healthy cells. The DNA aptamers seek out nucleoin and attach to the surface of the tumor. Then the nanobots penetrate the blood vessels that feed to the tumor and by unrolling release the thrombin molecules, which start clotting process that reduces blood flow to the tumor and starves the tumor (Murnane, 2018).

Anti-HIV nanobots: HIV virus destroys the immune system and thus the host become vulnerable to small diseases. By this process AIDS turns into a fatal disease. The HIV virus attacks WBCs and convert them to HIV. Thus the immune system fails and this is the reason for the death of the patient. There is no conventional drug that can cure this deadly disease. By usingnanobots, AIDS affected WBCs are converted to original form of WBC and thus maintain the constant amount of immune system (Bhuyan and Bardoloi, 2016).

\section{Host $\mathrm{WBC}+\mathrm{HIV}$ virus $=$ Infected $\mathrm{WBC}$}

Nanorobot + Infected WBC $=$ Restored WBC (Joshi and Pardeshi, 2013)

Surgery: Surgery is an invasive method and also liable for injury. It is also expensive and timeconsuming method whose success rate depends upon the skill and efficiency of the operating surgeon and his team. These limitations can be overcome by using nanobots (Eshaghian-Wilner, 2009). Surgically programmed nanobot can act as a semiautonomous onsite surgeon inside the body (Kshirsagar et al., 2014). When nanorobots treat the interior of the body, the controlling is preset to be done by the single machine itself thus ensuring much safer and exact treatment (Biswas and Sen, 2016). It would perform various functions such as detection of pathology, diagnosis, correcting lesions by nanomanipulation coordinated by an on-board computer (Manjunath and Kishore, 2014).

Neurosurgery: Spinal cord injury and nerve damage is an important topic of concern within the field of neurosurgery. Recently, different ways have been pursued with the goal of optimizing and improving nerve reconnection outcomes, including promoting the regeneration of axons via growth factors (Bregman et al., 2002) and enriched scaffolds (Chen et al., 2009). Restoring connectivity to transected axons is an inevitable step to the restoration function. However, this is limited by technical limitations of surgery (Chang et al., 2010).

Advancements in technology have led to the development of nanoscale device which allow manipulation of individual axons. A nanoknife with 40 nanometer diameter has been developed which is effective for axon surgery (Chang et al., 2007).The use of dielectrophoresis has been found to be effective in achieving controlled movement of axons within a surgical field (Sretavan et al., 2005). Following controlled transaction of axons and maneuvering them into position using dielectrophoresis, fusion between the two ends can be induced via electrofusion (berg, 1994), polyethylene glycol (Whittemore and Snyder, 1996) or laserinduced cell fusion (Steubing et al., 1991) amongst other methods.

One of the most effective ways to prevent morbidity and mortality in the field of neurosurgery is the treatment of cerebral aneurysm before rupture. About $10 \%$ patients die before arriving to the hospital, $5 \%$ die within 24 hours of aneurysm rupture and 50\% die within 30 days (Broderick et al., 1994). Nanobots can be used for screening a new aneurysm or closer monitoring of identified aneurysm. A design for an intravascular nanobot with the capability to detect aneurysm formation by detecting increased levels of nitric oxide synthase protein within the affected blood vessel has been proposed by 
Cavalcanti et al. (2009). These nanorobots can be given the capability of wirelessly communicate information about pertinent vascular changes to care providers, potentially decreasing screening costs of imaging and frequent follow up visits (Saadeh and Vyas, 2014).

S. Stupp and J. Kessler at Northwestern University in Chicago, designed small rod like nanofibers (Xu et al., 2002). This nanofiber was incorporated in nanobots called an amphiphiles and are capped with amino acids which incite the growth of neuron and ultimately prevent scar tissue formation (Chawla and Amiji, 2002).

Gene therapy: Medical nanobots can readily treat genetic diseases by comparing the molecular structures of both DNA and proteins found in the cell to desired reference structures (Sivasankar and Durairaj, 2012). Floating inside the nucleus of a human cell, an assembler built repair vessel performs some genetic maintenance. Stretching a super coil of DNA between its lower pair of robot arms, the nano machine gently pulls the unwound strand through an opening in its prow for analysis. Upper arms, meanwhile, detach regulatory proteins from the chain and place them in an intake port. The molecular structures of both DNA and proteins are compared to information stored in the database of a larger nano computer positioned outside the nucleus and connected to the cell-repair ship by a communications link. Irregularities found in either structure are corrected and the proteins reattached to the DNA chain, which re-coils into its original form with a diameter of only 50 nanometers, the repair vessel would be smaller than most bacteria and viruses, yet capable of therapies and cures well be beyond the reach of present-day physicians. "Internal medicine" would take on new significance (Adleman, 1995; Hamdi et al. 2008). Disease would be attacked at the molecular level and such maladies as cancer, viral infections and arteriosclerosis could be wiped out. Most human diseases involve a molecular malfunction at the cellular level, and cell function is largely controlled by gene expression and its resulting protein synthesis. One common practice of genetic therapy which has enjoyed only limited success is to supplement existing genetic material by inserting new genetic material into the cell nucleus, commonly using viral bacteriophage bacterial system cell plasmid/phospholipid microbubble cationic liposome, dendrimeric, chemical, nanoparticulate or other appropriate transfer vectors to breach the cell membrane (Freitas, 2007).

However, permanent gene replacement using viral carriers has largely failed thus far in human patients due to immune responses to antigens of the viral carrier as well as inflammatory responses, insertional mutagenesis and transient effectiveness. Excess gene copies, repeat gene clusters and partial trisomies and higher polysomies can often cause significant pathologies, sometimes mimicking aging. Attempting to correct excessive expression caused by these errors by implementing antisense transcription silencing on a whole-body, multigeneor wholechromosome basis would be far less desirable than developing more effective therapeutic methods that did not require such extensive remediation (Freitas, 2007).

Diabetes: At a typical glucose concentration, the nanobots try to keep the glucose levels ranging around $130 \mathrm{mg} / \mathrm{dl}$ as a target for the Blood Glucose Levels (BGLs). A variation of $30 \mathrm{mg} / \mathrm{dl}$ can be adopted as a displacement range, though this can be changed based on medical prescriptions. Medical nanobots can be designed in such a way that the significantly measured data can be transferred automatically through the radio frequency signals to the mobile phone carried by the patient and if the glucose achieves critical levels, the nanorobots emits an alarm through the mobile phone (Abhilash, 2010).

The SGLT3 (human sodium glucose cotransporter type 3) has influenced in regulating extracellular glucose concentration and define glucose level in the body and acts as sensor to identify glucose. According to Nandkishore et al. nanobots use chemo sensor that involve the modulation of SGLT3 glucosensor activity. By the help of this chemo sensor, nanobots determine the 
requirement of insulin and other treatment effectively (Kshirsagar et al., 2014).

Tetanus: Tetanus is caused by the pathogen Clostridium tetani that naturally present on the surface of the rusty nail and metallic object. When body surface is punctured by this rusty nail or metallic object, this bacterium may enter the body and release neurotoxin TeTx within a short period of time. This neurotoxin TeTx causes paralysis or locking of the whole body from head to foot leading to subsequent death. The conventional treatment is to inject anti-tetanus vaccine that counteract the $C$. tetani and neurotoxin TeTx within a short period of time. But it has several side effects like fever, redness and painful swelling around the injection site. In rare cases it causes brachial neuritis. An alternative treatment is to use programmable nanobot. When injected into the body, this nanobot will destroy the C. tetani and the released deadly neurotoxin TeTx and thus heal at a cellular level sparing the side effects of conventional vaccination (Nagalet al., 2012).

Myocardial infarction: The blood vessel blocking factors or plaque that is responsible for myocardial infarction can be detected and removed by nanobot molecule. The conventional treatment of myocardial infarction e.g. angioplasty depends on the surgical skills and sometimes has side effects but the process followed by nanobots is immune to these effects (Biswas and Sen, 2014).

Gout: When kidneys lose ability to remove waste from the breakdown of fats from the blood stream, this condition is called gout. This waste sometimes crystallizes at points near joints like knees and ankles and causes intense pain at these joints. A nanobot could break up the crystalline structures at the joints and provide relief from the symptoms (Strickland, 2010).

Alzheimer diseases: The amyloid- $\beta$ protein deposits show changes on gradients as a symptom of Alzheimer disease. This information serves for the early diagnosis of Alzheimer disease and to guide possible immunotherapy treatments, with more efficient neurotransmitters delivery, like dopamine and amino acids such as g-aminobutyrate (GABA), with better medical administration like nanobots (Cavalcanti et al., 2007).

Damaged tissue repair: Nanobots can easily repair and heal damaged tissue by taking existing molecules, replicating them and assembling new molecules into new layers of tissue. Nanorobots can slowly regrow portions of the damaged bone. It is hoped that one day, nanobot might be able to reproduce bone marrow. Other functional possibilities in the aspect of damaged tissue repair include: Closing of a split vein, reforming damaged skin, reducing dead flesh from a wound (Biswas and Sen, 2014).

Breaking up kidney stones: Nanorobots are used to break the kidney stones with the help of ultrasonic shocks. Kidney stones are painful and a large stone does not pass out in urine. Sometimes doctor break this stones by ultrasonic frequency, but these are not effective always. Nanorobots break up these kidney stones by using small laser and these smaller pieces are passing out in urine outside the body (Martinac and Metelko, 2005).

Skin diseases: A cream containing nanobots may be used to cure skin diseases. It removes the dead skin and excess oil, add missing oil, apply right amount of natural moisturizing compounds and participate in deep pore cleansing (Cavalcanti et al., 2004).

Inducing anesthesia: Nanobots may also be used as both general and local anesthesia. Being highly specific and target oriented, it reduces the anesthesia associated morbidity and mortality (Agarwal, 2012).

Body surveillance: By using nanobots, it is possible to monitor the vital organs and wireless transmission continuously which is not possible in case of conventional drug (Abeer, 2012). It will also give a quick response in case of sudden change in vital organs or warn against a possible risk e.g. high blood glucose in case of diabetes (Bhat, 2014).

Nanobot for detoxification: Self-propelled nanorobots have also been used as powerful detoxification tools with high cleaning capability. Similar to biosensing, detoxification strategies rely 
on self-propelled nanorobots that rapidly capture and remove the toxin to render the environment nontoxic. Efficient motion would facilitate the collision and binding of toxins to the motors, which are coated with desired functional materials. For example, nanomotors have been combined with cell-derived natural materials capable of mimicking the natural properties of their source cells toward novel nanoscale biodetoxification devices. Among different cell derivatives, red blood cells (RBCs) have shown excellent capability to function as toxin-absorbing nanosponges to neutralize and remove dangerous "pore-forming toxins" (PFTs) from the bloodstream (Hu et al., 2013). Motivated by the biological properties of RBCs, several different types of cellmimicking micromotors have been developed for detoxification. Wu et al. (2015a) presented a cellmimicking, water-powered micromotor based on RBC membrane- coated magnesium microparticles, which were able to effectively absorb and neutralize toxin in biological fluids (Wu et al., 2015a). Another detoxification strategy explored the combination of RBC membranes with ultrasound-propelled nanomotors as a biomimetic platform to effectively absorb and neutralize PFTs (Wu et al., 2015b).

Nanobots for targeted delivery: Drug delivery nanobots depend on systemic circulation and the force and navigation required for localized delivery and tissue penetration. To achieve precise delivery of therapeutic payloads to targeted disease sites, drug delivery vehicles are desired to have some unique capability that involve propelling force, controlled navigation and tissue penetration. The motor-like nanobots have the potential of rapidly transporting and delivering therapeutic payloads directly to disease sites and thus improve the therapeutic efficacy and reduce systemic side effects of highly toxic drugs (Li et al., 2017). A number of initial studies have been conducted to demonstrate the delivery function and performance of these nanobots in test tubes and in vitro environments. For example, $\mathrm{Wu}$ et al. (2015a) reported the preparation of a multilayer tubular polymeric nanomotor that is encapsulated by anticancer drug doxorubicin via a porous-membrane template-assisted layer-by-layer assembly. The nanomotor was able to deliver the loaded drug to the cancer cells (Wu et al., 2013). Ma et al. (2015) reported a chemically powered Janus nanomotor that functioned as an active nanoscale cargo delivery system and enabled a 100\% diffusion enhancement when compared with passive targeting without propulsion (Ma et al., 2015). SiRNA-loaded nanowires were shown to penetrate rapidly into different cell lines and noticeably improve the efficiency and speed of gene silencing process as compared with their static nanowire counterparts. Magnetic helical micro swimmers have also been used for targeted delivery of plasmid DNA (pDNA) to human embryonic kidney cells. The pDNA loaded motors were moved towards the cells and released their genetic cargo into the cells upon contact (Qiu et al., 2015).

Studies of in vivo activities of nanobots: Most of the studies have been performed in vitro but initial in vivo studies have already been performed and have demonstrated encouraging results. Recently Gao et al. (2015) conducted the very first in vivo study of chemically powered micromotors. The distribution, retention, cargo delivery ability and acute toxicity profile of the motors in a mouse's stomach have been carefully evaluated. The acid driven propulsion in the stomach enhanced the binding and retention of the motors in the stomach wall effectively considering zinc-based micro motor as a model. The micro motors gradually dissolve in the gastric acid, releasing their payloads and leaving nothing toxic behind (Gao et al., 2015).

The experiment of nanobots that destroy the tumor cells by preventing blood supply was performed on mice and pigs. The nanobots were injected intravenously into the blood stream of mice with breast cancer, melanoma, ovarian and lung cancer. The nanobots had located and surrounded the tumors within hours. Tissue damage that occurred due to blocking of blood supply was observed within $24 \mathrm{hrs}$. After $48 \mathrm{hrs}$ advanced blood clotting was observed and blood clot in all tumor was observed after 72 hrs. Nanobots were clearly successful in attacking the cancerous cells but if they could attack 
the healthy tissue the consequence would be more severe. But fortunately, such occurrence did not happen. Nanobots were also injected in mice and pig without tumors but no detectable change was found in normal blood coagulation and cell morphology. Such effectiveness of the treatment by nanobots against a variety of cancers holds a great hope for future (Murnane, 2018). There are two ways to remove the nanobots from the body. One, when nanobots task are completed they can be excreted from the body by human excretory channels. They can also be removed by active scavenger system (Requicha, 2003). Two, blood that is needed to be cleared may be passed from the patient to a specialized centrifugation apparatus. Here aural transmitters command nanobots to establish neutral buoyancy. No other solid blood component can maintain exact neutral buoyancy, hence those other components precipitate outward during gentle centrifugation and added back to filtered plasma on the other side of the apparatus. Meanwhile, after a period of centrifugation, the plasma that contains mostly suspended nanobots but few other solids, are drawn off through a 1micron filter, removing the nanorobots. Filtered plasma is recombined with centrifuged solid components and returned undamaged to the patient's body (Kharwade et al., 2013).

\section{Conclusion}

The recent invention of nanobots in the field of nanotechnology gives hope for the diagnosis and treatment of many hazardous diseases like cancer, heart disease, genetic disorder, HIV, diabetes with minimum side effects. It is also very much useful in the field of surgery and dentistry. Scientists are hopeful that, within next 10 years human blood will probably be streaming with tiny nanobots which will help to keep them away from getting sick. These will act on molecular level, protecting the biological system and thus ensuring a good and long life.

More research students are needed to develop the architecture and functions of nanobots and also to ensure its effectiveness and safety in human body.
This paper will help to provide a lot of information about nanobots which will help to perform these researches.

\section{Conflict of interest}

The authors declare that there is no conflict of interest.

\section{References}

Abeer, S. 2012. Future medicine: Nanomedicine. J. Int. Med. Sci. Acad. 25, 187-192.

Abhilash, M. 2010. Nanorobots. Int. J. Pharma. Bio. Sci. 1, 1-10.

Adleman, L.M. 1995. On constructing a molecular computer. DNA based computers. 27, 1-21.

Agarwal, A. 2012. The future of anaesthesiology. Indian J. Anaesth. 56, 524-526.

Berg, H. 1994. Methods in Enzymology: Membrane Fusion Techniques. (Düzgünes, N., Eds.), Academic Press, San Diego. 221, pp. 433-462.

Bhat, A.S. 2014. Nanobots: The future of medicine. Int. J. Manage Eng. Sci. 5, 44-49.

Bhuyan, M. and Bardoloi, S. 2016. Nanobots: A panacea to HIV. Int. Res. J. Eng. Tech. 3, 2390-2395.

Biswas, O. and Sen, A. 2016. Nanorobot the expected ever reliable future asset in diagnosis, treatment and therapy. In Foundations and Frontiers in Computer, Communication and Electrical Engineering: Proceedings of the 3rd International Conference C2E2, Mankundu, West Bengal, India. 15th-16th January, 2016. pp. 451.

Boonrong, P. and Kaewkamnerdpong, B. 2011. Canonical PSO based nanorobot control for blood vessel repair. World Acad. Sci. Eng. Technol. 58, 511-516.

Bregman, B.S., Coumans, J.V., Dai, H.N., Kuhn, P.L., Lynskey, J., McAtee, M. and Sandhu, F. 2002. Transplants and neurotrophic factors increase regeneration and recovery of function after spinal cord injury. Brain Res. 137, 257-273.

Broderick, J.P., Brott, T.G., Duldner, J.E., Tomsick, T. and Leach, A. 1994. Initial and recurrent bleeding are the major causes of death following subarachnoid hemorrhage. Stroke 25, 1342-1347.

Cavalcanti, A., Rosen, L., Kretly, L.C., Rosenfeld, M. and Einav, S. 2004. Nanorobotic challenges in biomedical applications, design and control. Electron. Circuits. Syst. 11, 447-450. 
Cavalcanti, A., Shirinzadeh, B., Freitas, R.A. and Kretly, L.C. 2007. Medical nanorobot architecture based on nanobioelectronics. Recent Pat. Nanotechnol. 1, 1-10.

Cavalcanti, A., Shirinzadeh, B., Fukuda, T. and Ikeda, S. 2009. Nanorobot for brain aneurysm. Int. J. Rob. Res. 28, 558-570.

Chang, W.C., Hawkes, E.A., Kliot, M. and Sretavan, D.W. 2007. In vivo use of a nanoknife for axon microsurgery. J. Neurosurg. 61, 683-692.

Chang, W.C., Hawkes, E., Keller, C.G. and Sretavan, D.W. 2010. Axon repair: Surgical application at a subcellular scale. Nanomed. Nanobiotechnol. 2, 151161.

Chawla, J.S. and Amiji, M.M. 2002. Biodegradable poly ( $\varepsilon$-caprolactone) nanoparticles for tumor targeted delivery of tamoxifen. Int. J. Pharmaceut. 249, 127138.

Chen, B.K., Knight, A.M., De Ruiter, G.C., Spinner, R.J., Yaszemski, M.J., Currier, B.L. and Windebank, A.J., 2009. Axon regeneration through scaffold into distal spinal cord after transection.J. neurotrauma. 26, 1759-1771.

Dietz, H., Douglas, S.M. and Shih, W.M. 2009. Folding DNA into twisted and curved nanoscale shapes. Science. 325, 725-730.

Douglas, S.M., Bachelet, I. and Church, G.M. 2012. A logic gated nanorobot for targeted transport of molecular payloads. Science. 335, 831-834.

Eshaghian-Wilner, M.M. 2009. Bio-inspired and nanoscale integrated computing (Vol. 1). John Wiley \& Sons, New Jersy, USA. pp. 1009-1012.

Freitas, R.A. 2005a. Current status of nanomedicine and medical nanorobotics. J. Comput. Theor. Nanosci. 2 , $1-25$.

Freitas, R.A. 2005b. Microbivores: Artificial mechanical phagocytes using digest and discharge protocol.J. Evol. Technol.14, 44-52.

Freitas, R.A. 2007. The ideal gene delivery vector: Chromallocytes, cell repair nanorobots for chromosome replacement therapy. J. Evol. Technol. 16, 90-97.

Gao, W., Dong, R., Thamphiwatana, S., Li, J., Gao, W., Zhang, L. and Wang, J. 2015. Artificial micromotors in the mouse's stomach: A step toward in vivo use of synthetic motors. ACS Nano. 9, 117-123.

Hamdi, M., Ferreira, A., Sharma, G. and Mavroidis, C. 2008. Prototyping bio-nanorobots using molecular dynamics simulation and virtual reality. Microelectron. J.39, 190-201.
Hu, C.M.J., Fang, R.H., Copp, J., Luk, B.T. and Zhang, L. 2013. A biomimetic nanosponge that absorbs pore forming toxins. Nat. Nanotechnol. 8, 336-339.

Joshi, A. and Pardeshi, A. 2013. Nanobot: An amazing invention in medical science. J. Electr. Electron. Eng.7, 84-90.

Kshirsagar, N., Patil, S., Kshirsagar, R., Wagh, A. and Bade, A. 2014. Review on application of nanorobots in health care. World J. Pharm. Pharm. Sci. 3, 472-80.

Kharwade, M., Nijhawan, M. and Modani, S. 2013. Nanorobots: A future medical device in diagnosis and treatment. Res. J. Pharm. Bio. Chem. Sci. 4, 12991307.

Kumar, R., Baghel, O., Sidar, S.K., Sen, P.K. and Bohidar, S.K. 2014. Applications of nanorobotics. Int. J. Sci. Res. Eng. Technol. 3, 1131-1137.

Li, J., De Ávila, B.E.F., Gao, W., Zhang, L. and Wang, J. 2017. Micro/nanorobots for biomedicine: Delivery, surgery, sensing, and detoxification. Sci. Robot. 2, 5-9.

Ma, X., Hahn, K. and Sanchez, S. 2015. Catalytic mesoporous Janus nanomotors for active cargo delivery. J. Am. Chem. Soc. 137, 4976-4979.

Manjunath, A. and Kishore, V. 2014. The promising future in medicine: Nanorobots. J. Biomed. Sci. Eng. 2, 4247.

Martinac, K. and Metelko, Z. 2005. Nanotechnology and diabetes. Diabetol. Croat. 34, 105-110.

Mishra, J., Dash, A.K. and Kumar, R. 2012. Nanotechnology challenges; nanomedicine; nanorabots. Int. Res. J. Pharmaceut. 2, 112-120.

Murnane, K. 2018. Nanorobots target and attack malignant tumors without harming healthy tissue. https://www.forbes.com/sites/kevinmurnane. Accessed on $29 / 03 / 2018$.

Nagal, D., Mehta, S.S., Sharma, S., Singh Mehta, G. and Mehta, H. 2012. Nanobots and their application in biomedical engineering. Proc. of the Int. Conf. on Advances in Electronics, Electrical and Computer Science Engineering. 3, 215-219.

Nanobot. Websters New Millennium ${ }^{\mathrm{TM}}$ Dictionary of English.

http://dictionar.reference.com/browse/nanobot. Retrieved January 12, 2018.

Nerlich, B. 2008. Powered by imagination: Nanobots at the science photo library. Sci. Culture. 17, 269-292.

Qiu, F., Fujita, S., Mhanna, R., Zhang, L., Simona, B.R. and Nelson, B.J. 2015. Magnetic helical microswimmers functionalized with lipoplexes for targeted gene delivery. Adv. Funct. Mater. 25, 16661671. 
Requicha, A.A. 2003. Nanorobots, NEMS, and nanoassembly. J. Electr. Electron. Eng. 91, 19221933.

Robert, A. F. J. 2009. Medical nanorobotics: The long term goal for nanomedicine. Nanomedicine Design of Particles, Sensors, Motors, Implants, Robots, and Devices (Mark, J.S. and Vesselin, N.S., Eds.), Artech House, Norwood MA. pp. 367-392.

Saadeh, Y. and Vyas, D. 2014. Nanorobotic applications in medicine: Current proposals and designs. Am. J. Robotic Surg. 1, 4-11.

Sahoo, S.K., Parveen, S. and Panda, J.J. 2007. The present and future of nanotechnology in human health care. Nanomedicine. 3, 20-31.

Sharples, L. 2011. Nanotechnology in dentistry: Developing new materials, non invasive treatments and the ethical issues involved in nanotechnology. Patho. Lec. S. Medi. 10, 50-57.

Sivasankar, M. and Durairaj, R. 2012. Brief review on nano robots in bio medical applications. Adv.Robotics Automa. 1, 2-5.

Sretavan, D.W., Chang, W., Hawkes, E., Keller, C. and Kliot, M. 2005. Microscale surgery on single axons. $J$. Neurosurg. 57, 635-646.

Steubing, R.W., Cheng, S., Wright, W.H., Numajiri, Y. and Berns, M.W. 1991. Laser induced cell fusion in combination with optical tweezers: The laser cell fusion trap. Cytometry. B. Clin. Cytom. 12, 505-510.

Strickland, J. 2010. How nanorobots will work. How Stuff Works. 2, 14-18. https://electronics.howstuffworks. com/nanorobot.htm.
Sujatha, V., Suresh, M. and Mahalaxmi, S. 2010. Nanorobotics: A futuristic approach. Univ. J. Dent. Sci.1, 86-90.

Uriarte, S. L. 2011. Nanorobots. Technical report Escuela Superior De Ingenieros De Bilbao, BilbokoIngeniarienGoiEskola, Universidad Del País Vasco / Euskal Herriko Unibersitatea. http://nanobio.ehu.es/files/nanorobots.

Whittemore, S.R. and Snyder, E.Y. 1996. Physiological relevance and functional potential of central nervous system derived cell lines. Mol. Neurobiol. 12, 13-38.

Wu, Z., Wu, Y., He, W., Lin, X., Sun, J. and He, Q. 2013. Self propelled polymer based multilayer nanorockets for transportation and drug release. Angew. Chem. Int. Ed. Engl. 52, 7000-7003.

Wu, Z., Li, J., de Ávila, B.E.F., Li, T., Gao, W., He, Q., Zhang, L. and Wang, J. 2015a. Water powered cell mimicking janusmicromotor. Adv. Funct. Mater. 25, 7497-7501.

Wu, Z., Li, T., Gao, W., Xu, T., Jurado, Sánchez, B., Li, J., Gao, W., He, Q., Zhang, L. and Wang, J. 2015b. Cell membrane coated Synthetic nanomotors for effective biodetoxification. Adv. Funct. Mater. 25, 3881-3887.

Xu, L., Frederik, P., Pirollo, K.F., Tang, W.H., Rait, A., Xiang, L.M., Huang, W., Cruz, I., Yin, Y. and Chang, E.H. 2002. Self assembly of a virus mimicking nanostructure system for efficient tumor targeted gene delivery. Hum. Gene Ther. 13, 469-481. 